\title{
Pharmacology of pain
}

\section{Fornasari ${ }^{1}$, S. Coaccioli ${ }^{2}$}

1Department of Medical Biotechnologies and Translational Medicine, University of Milan; 2Internal Medicine and Rheumatology Unit, Perugia University School of Medicine, District of Terni, Santa Maria General Hospital, Terni, Italy

\section{SUMMARY}

This article discusses the mechanisms of action of the main drugs used to treat pain, in particular inflammatory pain. The drugs are described following a classification based on the steps of pain processing that they primarily affect.

Key words: Pain, Pharmacology, Inflammatory pain.

\section{INTRODUCTION}

M ore than $50 \%$ of the patients suffering from chronic pain complain that they receive inadequate relief from pharmacological treatments. It is widely accepted that, unless adequately and rapidly treated, chronic pain can become an often intractable and possibly irreversible disease in itself. This is mainly due to the neuroplastic changes in the pain pathways, i.e. alterations caused by their use or modulatory influences. Such changes may be acute or chronic, and occur at molecular, synaptic, cell and network levels (1). They also have their own clinical counterparts, as the pain acquires new semeiotic features: it can be spontaneous, evoked by innocuous and normally non-painful stimuli (allodynia), or perceived as being more intense and longer lasting than usual when painful stimuli are applied (hyperalgesia). As chronic pain is very complex and involves a number of pathogenetic mechanisms, a multi-target pharmacological treatment is probably the best approach, because it allows more than one target to be attacked at a time and to use low doses of the individual active ingredients (2).

Corresponding author: Diego Fornasari Department of Medical Biotechnology and Translational Medicine University of Milan Via Vanvitelli, 32 - 20129 Milan, Italy E-mail: diego.fornasari@unimi.it

\section{DRUGS FOR THE TREATMENT OF CHRONIC PAIN} the definition of chronic pain implies that
In the case of non-malignant conditions, it persists for longer than three months. It may be nociceptive, neuropathic or functional, and all forms share some common characteristics $(3,4)$.

Pain processing is organized in five essential steps: transduction, conduction, modulation, transmission and perception. Transduction is the process that converts mechanic, thermic (cold or heat), and chemical (low or high $\mathrm{pH}$ ) energies with high intensity, and thus potentially dangerous, in an electrical signal, the generator potential, that, in turn, will trigger the onset of the action potential. This occurs in the peripheral terminals of the primary afferent somatic and visceral nociceptive fibers that have their bodies in the dorsal root ganglia. These high-threshold primary sensory neurons that are specialized in responding to strong and potentially tissue-damaging stimuli are known as nociceptors. Their peripheral terminals contain highly specialized transducer proteins, most of which are channels that open in response to specific high-energy stimuli, thus becoming permeable to the calcium and sodium ions that are responsible for terminal depolarization. Most of these transducers have been characterized at molecular level, and their function has immediately appeared to be more complex than expected. For example, the transducer TRPV1 (a member of the transient receptor potential family) (5) becomes activated in response to heat exceeding $43^{\circ} \mathrm{C}$, but also in response to vanilloid 
chemical ligands, such as capsaicin, the pungent ingredient of chili peppers.

One possible mechanism underlying the use of capsaicin in pain control is related to its effects on the transmembrane potential of nociceptive fibers, which is progressively lost because the sustained opening of the TRPV1 channel makes it no longer excitable.

Pain neural structures are rapidly modified in the case of acute and, especially, chronic inflammatory pain, during which the activation thresholds of peripheral nociceptors is lowered and their responsiveness is increased. This peripheral sensitization is mainly the result of the phosphorylation of two molecular structures peripherally involved in pain processing: the transducer TRPV1 and the voltage-gated sodium ion Nav 1.8 channel. Upon phosphorylation, TRPV1 becomes activated at $37^{\circ} \mathrm{C}$ instead of $43^{\circ} \mathrm{C}$, and the lower activation threshold of Nav 1.8 allows the passage of a larger amount of sodium ions when it opens. The phosphorylation of these substrates is due to the activation of different kinases by sensitizing agents that work through their own membrane receptors, such as prostaglandins, bradykinin and adenosine, which are released by inflammatory cells.

Peripheral sensitization is an important target for pain pharmacology, because non-inflammatory anti-rheumatic drugs (NSAIDs) are mainly used to reduce simply the peripheral presence of prostaglandins. Steroids are also used to decrease the synthesis of sensitizing molecules.

If the inflammatory process underlying pain resolves in a few days, all of the plastic modifications in the nociceptors revert to normality. On the contrary, persistent inflammation (or any other pathological condition that causes chronic pain, such as peripheral neuropathic pain) provokes a repetitive high-intensity synaptic transmission, which leads to plastic changes in the spinal cord that not only affect second-order spinothalamic neurons, but also interneurons, some of which may even die as a result of the abnormal peripheral stimulation. This is also known as central sensitization.
Conduction is the step in which action potentials travel along axons to reach the spinal cord. For conduction to occur, voltage-gated sodium ion channels must convert the generator potential at the peripheral terminals into an action potential. Various isoforms of voltage-gated sodium ion channels are known, but Nav 1.8 and Nav 1.9 are selectively expressed in nociceptors, and are blocked by local anesthetic and anti-epileptic drugs. Nociceptors can have unmyelinated (C-fiber) or thinly myelinated (A $\delta$-fiber) axons. C-fibers are probably more involved in chronic pain. When action potentials reach the nociceptor terminals in the dorsal horns of the spinal cord, the N-type voltage-gated calcium channels open and promote the neurotransmitter release. N-type calcium channels contain the $\alpha 2 \delta$ subunit to which pregabalin and gabapentin bind, thus blocking the influx of calcium ions and the neurotransmitter release (6). This is the pharmacological property that makes them useful in the treatment of neuropathic pain.

The transmission step coincides with the synaptic communication between the firstand second-order neurons. The synapse between a nociceptor (or first-order neuron) and a second-order spinothalamic neuron is primarily glutamatergic. In the case of acute nociceptive pain, glutamate mainly binds to AMPA receptors, which are ligand-gated ion channels that are highly permeable to sodium ions, which depolarize second-order neurons and trigger the action potential reaching the thalamus. More intense or sustained C-fiber nociceptor activation leads to the release of neuropeptide modulators (substance P) and sustained post-synaptic depolarization. This depolarization removes the voltage-dependent, $\mathrm{Mg}^{2+}$-induced blockade of NMDA receptors, the other class of glutamate receptors expressed in second-order neurons of the dorsal horn, thus causing a large influx of $\mathrm{Ca}^{2+}$. NMDA receptor activation is an essential step in central sensitization that always accompanies chronic pain. The involvement of NMDA receptors in chronic pain justifies the use of drugs that block them, such as ketamine (7). 
Although it is believed that prostaglandins are mainly involved in peripheral sensitization, new data indicates that they can be synthesized and released in the spinal cord by neuronal and glial cells, and therefore participate in central sensitization (8). It has been recently shown that peripheral inflammation increases the synthesis of spinal prostacyclin, and that this induces the c-AMP-dependent translocation of NMDA receptors to the post-synaptic membranes of second-order neurons. Up-regulation of prostacyclin synthesis in the spinal cord is seen as early as one hour after the induction of peripheral inflammation, thus suggesting a functional role during the onset of central sensitization (9). Taken together, this bulk of data extends the contribution of NSAIDs to the treatment of inflammatory pain.

The release of synaptic vesicles at the spinal cord synapse is finely controlled by various mechanisms. For example, some of the TRP channels present in the periphery are also expressed in the dorsal horn at pre-terminal and pre-synaptic level, where they modulate the release of neurotransmitters, such as TRPA1, that can increase glutamate release, and thus participate in the mechanisms of central sensitization mechanisms. It is believed that NAPQI mediates the well-known hepato- and nephrotoxic effects of paracetamol. However, NAPQI metabolites can also be detected in human and mouse blood or urine after the ingestion of non-toxic, therapeutic doses of paracetamol. There is now evidence that NAPQI and other paracetamol metabolites can bind to TRPA1 and, like capsaicin in the case of TRPV1, induce a progressive reduction in transmembrane potential, with an initial depolarization block and the sustained inhibition of sodium and calcium currents that negatively affect the neurotransmitter release (10). Paracetamol therefore has an additional and particular mechanism of action that makes it an excellent partner in the multi-target pharmacological treatment of chronic pain.

Not all impulses coming from the periphery reach the thalamus, because synaptic transmission in the spinal cord is also regulated by the action of local interneurons and projections that descend from the brainstem to the dorsal horn. The major inhibitory neurotransmitters in the dorsal horn are opioids, norepinephrine, serotonin, glycine and GABA. The role of norepinephrine and serotonin in the spinal modulation of pain stimuli explains why antidepressant drugs that inhibit their re-uptake are used to treat neuropathic pain (11). Opioids induce analgesia because of their action in the brain, brainstem, spinal cord and, under some circumstances, the peripheral terminals of primary afferent neurons. All endogenous opioid peptides (which include $\beta$-endorphin, the enkephalins and the dynorphins) bind to seven transmembrane $\mathrm{G}$ protein-coupled receptors, which are traditionally divided into three classes: $\mu, \delta$ and $\kappa(12)$.

All opioid receptors are coupled to inhibitor $\mathrm{G}$ proteins, and receptor activation inhibits adenylate cyclase and the intracellular generation of cAMP. However, it is believed that their coupling to $\mathrm{K}^{+}$and $\mathrm{Ca}^{2+}$ ion channels is the most important mechanism by which both endogenous and exogenous opioids induce analgesia. In particular, the overwhelming majority of opioid receptors in the dorsal horn of the spinal cord are $\mu$ receptors: these have a predominantly pre-synaptic location (more than $70 \%$ ) on the central terminals of nociceptors $(\mathrm{C}$ and A $\delta$ fibers), whereas the remaining 30\% are located post-synaptically on dendrites of second-order spinothalamic neurons and interneurons. The endogenous opioids $\beta$-enkefalin and endorphins that act on the $\mu$ receptors in the dorsal horn of the spinal cord are predominantly released by interneurons, whose activation depends on the activity of the descending pathways. The activation of pre-synaptic $\mu$ receptors inhibits calcium ion channels and prevents the release of neurotransmitters. The activation of post-synaptic $\mu$ receptors activates potassium ion channels, leading to an efflux of potassium ions and the hyperpolarization of the projecting cell. The stimulation of $\mu$ opioid receptors in the spinal cord is therefore a very effective means of blocking synaptic transmission, thus limiting the number of nociceptive stimuli that 
reach the thalamus and eventually the cortex where the conscious perception of pain occurs.

\section{CONCLUSIONS}

It is widely accepted that there is a need for new molecules with innovative pharmacodynamics and pharmacokinetics in order to fight pain, which remains a very serious burden for patients, families, physicians and communities. However a better understanding of the mechanisms of action of the available drugs may increase their efficacy as a result of a more appropriate clinical use, alone or especially in combination, which may be the best way to treat pain.

\section{REFERENCES}

1. Kuner R. Central mechanisms of pathological pain. Nat Med. 2010; 16: 1258-66.

2. Chaparro LE, Wiffen PJ, Moore RA, Gilron I. Combination pharmacotherapy for the treatment of neuropathic pain in adults. Cochrane Database Syst Rev. 2012; 7: CD008943.

3. Fornasari D. Pain mechanisms in patients with chronic pain. Clin Drug Investig. 2012; 32: 45-52.

4. Woolf CJ. Pain: moving from symptom con- trol toward mechanism-specific pharmacologic management. Ann Intern Med. 2004; 140: 441-51.

5. Patapoutian A, Tate S, Woolf CJ. Transient receptor potential channels: targeting pain at the source. Nat Rev Drug Discov. 2009; 8: 55-68.

6. Stahl SM, Porreca F, Taylor CP, Cheung R, Thorpe AJ, Clair A. The diverse therapeutic actions of pregabalin: is a single mechanism responsible for several pharmacological activities? Trends Pharmacol Sci. 2013; 34: 332-9.

7. Youn DH, Gerber G, Sather WA. Ionotropic glutamate receptors and voltage-gated $\mathrm{Ca}^{2}+$ channels inlong-term potentiation of spinal dorsal horn synapses and pain hypersensitivity. Neural Plast. 2013; 2013: 654257.

8. Burian M, Geisslinger G. COX-dependent mechanisms involved in the antinociceptive action of NSAIDs at central and peripheral sites. Pharmacol Ther. 2005; 107: 139-54.

9. Schuh CD, Brenneis C, Zhang DD, Angioni C, Schreiber Y, Ferreiros-Bouzas N, et al. Prostacyclin regulates spinal nociceptive processing through cyclic adenosine monophosphateinduced translocation of glutamate receptors. Anesthesiology. 2013 [Epub ahead of print].

10. Andersson DA, Gentry C, Alenmyr L, Killander D, Lewis SE, Andersson A, et al. TRPA1 mediates spinal antinociception induced by acetaminophen and the cannabinoid $\Delta$ (9)-tetrahydrocannabiorcol. Nat Commun. 2011; 2: 551.

11. Hartrick CT. Noradrenergic reuptake inhibition in the treatment of pain. Expert Opin Investig Drugs. 2012; 21: 1827-34.

12. Pathan H, Williams J. Basic opioid pharmacology: an update. Br J Pain. 2012; 6: 11-6. 